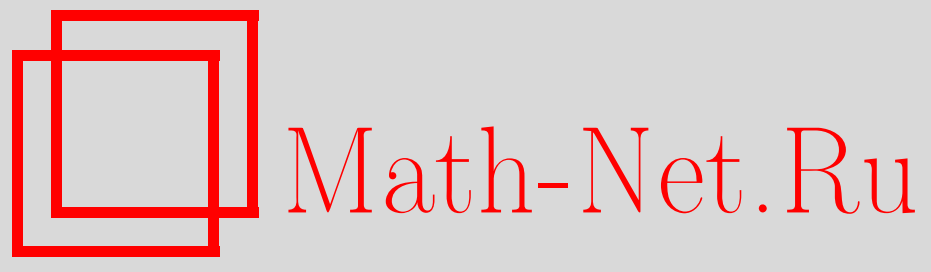

Э. Б. Винберг, Об автоморфных формах на симметрических областях типа IV, УМH, 2010, том 65, выпуск 3, 193-194

DOI: https://doi.org/10.4213/rm9343

Использование Общероссийского математического портала Math-Net.Ru подразумевает, что вы прочитали и согласны с пользовательским соглашением http://www . mathnet.ru/rus/agreement

Параметры загрузки:

IP : 52.87 .193 .239

26 апреля 2023 г., 16:51:12

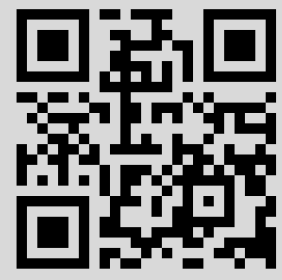




\section{Об автоморфных формах на симметрических областях типа IV}

\section{Э. Б. Винберг}

Симметрической областью типа IV называется эрмитово симметрическое пространство $\mathscr{D}_{n}=\mathrm{O}_{2, n}^{+} /\left(\mathrm{SO}_{2} \times \mathrm{O}_{n}\right)$, где $\mathrm{O}_{2, n}^{+}-$подгруппа индекса 2 псевдоортогональной группы $\mathrm{O}_{2, n}$, выделяемая условием положительности левого верхнего минора порядка 2. Она моделируется областью на квадрике в проективизации $(n+2)$-мерного комплексного векторного пространства, в котором естественным образом действует группа $\mathrm{O}_{2, n}$. Тем самым определено $\mathrm{O}_{2, n}^{+}$-эквивариантное линейное расслоение $\pi: \mathscr{L}_{n} \rightarrow \mathscr{D}_{n}$ ( $n$-я степень которого есть каноническое расслоение).

Пусть $Г$ - дискретная подгруппа конечного кообъема в группе $\mathrm{O}_{2, n}^{+}$. Для любого неотрицательного целого $k$ рассмотрим Г-инвариантные голоморфные функции на $\mathscr{L}_{n}$, однородные степени $-k$ на каждом слое. При $n \geqslant 3$ они образуют конечномерное векторное пространство $A\left(\mathscr{D}_{n}, \Gamma\right)_{k}[1]$. Алгебра $A\left(\mathscr{D}_{n}, \Gamma\right)=\sum_{k=0}^{\infty} A\left(\mathscr{D}_{n}, \Gamma\right)_{k}$ называется естественной алгеброй автоморфных форм на $\mathscr{D}_{n}$ относительно группы $\Gamma$, а элементы пространства $A\left(\mathscr{D}_{n}, \Gamma\right)_{k}$ называются автоморфными формами веса $k$.

Известно [1], что $A\left(\mathscr{D}_{n}, \Gamma\right)$ - нормальная конечно порожденная градуированная алгебра. Аффинное многообразие $\operatorname{Spec} A\left(\mathscr{D}_{n}, \Gamma\right)$ содержит аналитический фактор $\mathscr{L}_{n} / \Gamma$ как открытое по Зарискому подмножество, граница которого не более чем двумерна.

Пусть теперь $\Gamma_{n}=\mathrm{O}_{2, n}^{+}(\mathbb{Z})$. В 1962 г. Игуза [2] доказал, что для некоторой подгруппы $\Gamma \subset O_{2,3}^{+}$, соизмеримой с $\Gamma_{3}$, алгебра $A\left(\mathscr{D}_{3}, \Gamma\right)$ свободно порождается формами весов $4,6,10,12$. Главным результатом настоящей работы является следующая теорема.

Теорема 1. При $n \in\{4,5,6,7\}$ алгебра $A\left(\mathscr{D}_{n}, \Gamma_{n}\right)$ свободно порождается формами весов, указанных в следующей таблице:

\begin{tabular}{l|l}
$n$ & Веса \\
\hline 4 & $4,6,8,10,12$ \\
5 & $4,6,8,10,12,18$ \\
6 & $4,6,8,10,12,16,18$ \\
7 & $4,6,8,10,12,14,16,18$
\end{tabular}

СледСтвие 1. При $n \in\{4,5,6,7\}$ фактор $\mathscr{L}_{n} / \Gamma_{n}$ является односвязным комплексным многообразием.

Это означает, что группа $\Gamma_{n}$ как группа преобразований многообразия $\mathscr{L}_{n}$, а также стабилизатор любой точки этого многообразия порождается (комплексными) отражениями. Соответствующая группа голоморфных преобразований области $\mathscr{D}_{n}$ также порождается отражениями.

Для любого $n \geqslant 3$ естественные вложения $\mathscr{L}_{n} \hookrightarrow \mathscr{L}_{n+1}$ и $\Gamma_{n} \hookrightarrow \Gamma_{n+1}$ индуцируют замкнутое вложение $\mathscr{D}_{n} / \Gamma_{n} \hookrightarrow \mathscr{D}_{n+1} / \Gamma_{n+1}$ и, тем самым, эпиморфизм градуированных алгебр $A\left(\mathscr{D}_{n+1}, \Gamma_{n+1}\right) \rightarrow A\left(\mathscr{D}_{n}, \Gamma_{n}\right)$. При $n=4,5,6$ ядром этого эпиморфизма является главный идеал алгебры $A\left(\mathscr{D}_{n+1}, \Gamma_{n+1}\right)$, порожденный образующей веса 18 , 16, 14 соответственно.

Доказательство теоремы основано на интерпретации фактора $\mathscr{D}_{n} / \Gamma_{n}$ как пространства модулей кратно поляризованных поверхностей типа $K 3$.

А именно, пусть $\mathbb{R}^{3,19}$ - псевдоевклидово векторное пространство с ортогональным базисом $\left\{e_{0}, e_{1}, \ldots, e_{21}\right\}$, в котором $\left(e_{0}, e_{0}\right)=\left(e_{20}, e_{20}\right)=\left(e_{21}, e_{21}\right)=1$, а квадраты прочих базисных векторов равны -1 . Пусть $J$ - решетка в $\mathbb{R}^{3,19}$, порожденная всеми целочисленными векторами с четной суммой координат и вектором

Работа выполнена при поддержке РФФИ (грант № 07-01-00390). 
$\rho=\left(e_{0}+e_{1}+\cdots+e_{21}\right) / 2$. Это четная унимодулярная квадратичная решетка. Известно, что группа гомологий $H_{2}(X, \mathbb{Z})$ гладкой поверхности $X$ типа $K 3$, снабженная формой пересечения $(\cdot, \cdot)$, изоморфна $J$. Группа $S(X)$ алгебраических циклов поверхности $X$ является примитивной подрешеткой сигнатуры $(1, m)$ в $J$.

Пусть $S_{n}(3 \leqslant n \leqslant 7)$ - пересечение решетки $J$ с линейной оболочкой векторов $e_{0}, e_{1}, \ldots, e_{19-n}$. Рассмотрим поверхности типа $K 3$, группа алгебраических циклов которых содержит $S_{n}$, и снабдим их поляризацией $h=4 e_{0}+e_{1}+\cdots+e_{12}$ с квадратом 4 . Пространство модулей таких поверхностей обозначим через $M_{n}$. При $n \neq 6$ отображение периодов устанавливает изоморфизм аналитических пространств $M_{n} \simeq \mathscr{L}_{n} / \Gamma_{n}$. Что касается пространства $M_{6}$, то оно изоморфно фактору области $\mathscr{D}_{6}$ по некоторой надгруппе индекса 3 группы $\Gamma_{6}$; для перехода к фактору по $\Gamma_{6}$ нужно рассматривать поверхности с дополнительной структурой, о которой будет сказано ниже.

С другой стороны, можно показать, что для любой поверхности $X \in M_{n}$ решетка $S(X)$ не содержит изотропных векторов $u$ с $(h, u)=1$ или 2 . В силу известного критерия [1] это означает, что линейная система $|h|$ задает бирациональный изоморфизм поверхности $X$ на квартику $Y \subset \mathbb{C P}^{3}$, имеющую не более чем простые особенности. При $n \in\{4,5,6,7\}$ получаемые таким образом квартики характеризуются тем, что они имеют особую точку $о$, через которую проходят две плоскости $\Pi_{1}$ и $\Pi_{2}$, пересекающиеся по некоторой прямой $l \subset Y$; при этом $\Pi_{1}$ пересекает $Y$ по четверной прямой $l$, а $\Pi_{2}-$ по прямой $l$ и, кроме того,

(а) при $n=7$ - по кривой второго порядка, касающейся $l$ в точке $o$, и прямой, проходящей через точку $о$;

(b) при $n=6$ - по трем прямым, проходящим через точку $o$; для получения фактора по группе $\Gamma_{6}$ следует считать одну из этих прямых выделенной;

(c) при $n=5$ и $n=4-$ по прямой и двойной прямой $m$, проходящим через точку $o$; случай $n=4$ выделяется условием, что прямая $m$ содержит вырожденную особую точку, отличную от 0 .

Это позволяет описать пространство $M_{n}$ как фактор по группе $\mathrm{SL}_{4}(\mathbb{C})$ некоторого инвариантного алгебраического подмногообразия $P_{n}$ в пространстве квартик и тем самым установить изоморфизм между алгеброй $A\left(\mathscr{D}_{n}, \Gamma_{n}\right)$ и алгеброй $\mathrm{SL}_{4}(\mathbb{C})$-инвариантных многочленов на конусе над $P_{n}$. Путем построения сечения удается доказать, что последняя алгебра изоморфна алгебре инвариантов максимального тора группы $\mathrm{SL}_{4}(\mathbb{C})$ в некотором $(n+4)$-мерном подпространстве пространства $\mathrm{S}^{4}\left(\left(\mathbb{C}^{4}\right)^{*}\right)$, которая оказывается свободной с образующими степеней, указанных в таблице.

Я благодарен Э. Лойенге, В. В. Никулину и Ю. Г. Прохорову за ценные консультации. Работа была закончена во время моего пребывания в университете Билефельда в июле-августе 2009 г., финансировавшегося Немецким обществом естествоиспытателей.

\section{Список литературы}

[1] E. Looijenga, "Compactifications defined by arrangements, II: Locally symmetric varieties of type IV", Duke Math. J., 119:3 (2003), 527-588. [2] J.-I. Igusa, "On Siegel modular forms of genus two", Amer. J. Math., 84:1 (1962), 175-200.

Э. Б. Винберг (亡̀. В. Vinberg)

Московский государственный университет им. М. В. Ломоносова

E-mail: vinberg@zebra.ru
Представлено В. М. Бухштабером Принято редколлегией 24.01 .2010 